\title{
Evaluation of Refraction Effects in Dry Medical Microwave Imaging Setups
}

\author{
Daniela M. Godinho, João M. Felício, Member, IEEE, Carlos A. Fernandes, Senior Member, IEEE, and Raquel C. \\ Conceição
}

\begin{abstract}
Dry Microwave Imaging (MWI) systems are more practical, hygienic and fast to operate since they do not require immersion liquid. However, the dielectric contrast between air and the part of the body under examination is larger, causing larger refraction effects. Including refraction in the image reconstruction algorithm significantly increases the computational effort, especially when imaging non-uniform shapes. Hence, our systematic study aims to evaluate the impact of neglecting refraction effects on MWI by using quantitative metrics and define objective guidelines that are lacking in the literature. We perform comparative studies with a spherical numerical phantom (which is typically used to represent simplified breast or head phantoms) by varying the phantom relative permittivity values between 4 and 40, metallic targets diameter between 5 and $15 \mathrm{~mm}$, and the number of probing antennas. Additionally, the refraction effects are evaluated with anthropomorphic body phantoms representing a breast and the axillary region. We numerically and experimentally show that refraction tends to have greater impact on imaging results when phantom relative permittivity values exceed 8 , while it has a minor effect in the remaining tested cases. This favours potential fast real-time image reconstruction. This study provides useful criteria to decide whether refraction should be considered or not for imaging reconstruction when developing new dry medical MWI setups.
\end{abstract}

Index Terms-Dry microwave imaging setup, medical microwave imaging, refraction, ultrawide-band radar.

\section{INTRODUCTION}

$\mathbf{R}$ ECENT medical Microwave Imaging (MWI) studies have presented the use of dry imaging prototypes where the antennas are placed a few centimetres away from the skin in air [1]-[6]. Dry and contactless setups prevent hygienerelated issues and are easier to maintain compared to setups that use coupling medium, at a cost of larger skin backscatter response. Yet, it has been recently demonstrated that the response of breast tumours is not significantly affected by the increased air/skin reflection [1]. Due to the dielectric contrast

Manuscript submitted on January 15, 2021. This work is supported by Fundação para a Ciência e a Tecnologia - FCT under fellowship SFRH/BD/129230/2017, FCT/MEC (PIDDAC) under the Strategic Programme UIDB/00645/2020, and also in part by FEDER-PT2020 Partnership Agreement under Grant UIDB/EEA/50008/2020.

Daniela M. Godinho and Raquel C. Conceição are with Instituto de Biofísica e Engenharia Biomédica, Faculdade de Ciências da Universidade de Lisboa, 1749-016 Lisbon, Portugal (e-mail: dgodinho94@gmail.com, raquelcruzconceicao@gmail.com).

João M. Felício is with Centro de Investigação Naval (CINAV), Escola Naval, Almada, Portugal, and with Instituto de Telecomunicações, Instituto Superior Técnico, Universidade de Lisboa, Lisbon, Portugal (e-mail: joao.felicio@lx.it.pt).

Carlos A. Fernandes is with Instituto de Telecomunicações, Instituto Superior Técnico, Universidade de Lisboa, Lisbon, Portugal (e-mail: carlos.fernandes@1x.it.pt). in the air/body interface, the rays change their direction of propagation. Some authors have presented algorithms with refraction calculation validated in regular shapes [7], [8], but the impact in terms of image improvement and computational time added when refraction is calculated has not been assessed. Nevertheless, most medical dry MWI setups still assume direct path ray propagation and no one has yet quantified the impact this assumption may have on resulting images. The computation of the refracted rays can be computationally heavy and time consuming, especially when imaging irregular body parts, which hinder real-time image reconstruction. As a result, it is relevant to determine the conditions where refraction does not substantially improve the imaging results so that refraction can be discarded when reconstructing microwave medical images.

Recently, we presented a preliminary study [9] using a spherical breast phantom with relative permittivity of 8 and, qualitatively, we did not observe a significant improvement when including refraction to imaging results. In this paper, we substantially extend our study with the spherical breast phantom, analysing the influence of three parameters which may influence the impact of refraction: relative permittivity of the phantom, targets size, and number of probing antennas. Moreover, we evaluate the refraction effects in more realisticshaped body parts (breast and axillary region) in simulated and experimental data, in order to assess whether the body shape influences the impact of refraction effects. Finally, we provide criteria which can be used by other researchers to decide whether refraction calculation can be avoided with no impact on image quality, while potentially allowing a faster and less computationally intensive computation.

\section{IMAGE RECONSTRUCTION ALGORITHM}

The imaging algorithm is based on wave migration [9], [10]. It receives as inputs both the signal recorded by each antenna $\left(\mathbf{s}_{a a}\right)$ and the distances from each voxel to the antenna. The only difference in the algorithm when refraction is and is not considered is in the distances calculation. These distances correspond to the Euclidean distances travelled in air between antenna $a$ and entry point on the phantom $i\left(d_{a i}\right)$ and in the dielectric between interface point $i$ and voxel $v\left(d_{i v}\right)$ (Fig. 1a).

When refraction is disregarded, the entry point on the phantom is calculated as the intersection point on the phantom surface assuming straight ray path; this is the common procedure reported in the literature. When refraction is considered, the entry point is calculated assuming the directions of the incident and refracted ray as defined by Snell's law: 


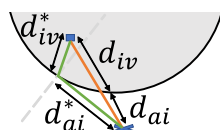

(a)

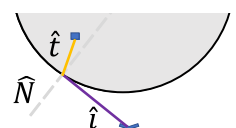

(b)

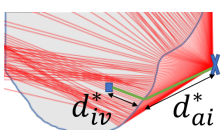

(c)
Fig. 1. Schematics for refraction calculation with $(a, b)$ analytical form and (c) ray tracing algorithm. The distances travelled in air (white background) and in dielectric (grey background) when refraction is not considered are $d_{a i}$ and $d_{i v}$, and when it is considered are $d_{a i}^{*}$ and $d_{i v}^{*}$, respectively.

$\left(\frac{1}{n_{\text {diel }}} \hat{i}-\hat{t}\right) \times \hat{N}=0$, where $\hat{i}, \hat{t}$ and $\hat{N}$ are unitary vectors in the direction of the incident ray, of the refracted ray and normal to the surface, respectively (Fig. 1b). Since the computation of the normal vector is not always analytically possible depending on the shape of the body, we considered two methods to calculate the refracted ray:

1) Analytical form: For a spherical surface, the $\hat{N}$ vector is easily determined. In this case, Snell's law returns a single analytical solution for the entry point coordinates in the sphere, after excluding imaginary or physically impossible solutions.

2) Ray tracing algorithm: For irregular shapes, refraction is computed locally following the ray paths (Fig. 1c). Given a $2 \mathrm{D}$ contour of the phantom and the coordinates of a given antenna as origin, several incident rays are traced towards the body. The direction of the refracted rays are locally calculated with Snell's law at the intersection point between each incident ray and the phantom, assuming $n_{\text {diel }}$. The distances $d_{a i}$ and $d_{i v}$ are calculated from the rays that intersect each voxel, $v$. When multiple refracted rays intersect a voxel $v$ for the same antenna position, the intensity of the voxel is calculated as the contribution of the shortest ray path.

\section{A. Performance metrics}

The refraction effects in the reconstructed images are evaluated comparing common metrics used to assess the quality of target detection: Signal-to-Clutter Ratio (SCR), Full Width Half Maximum (FWHM) and Location Error (LE). Two other metrics are used to evaluate how refraction computation affects the images: Maximum Magnitude Ratio (MMR) and Distance Error (DE). We consider the SCR threshold to classify as a positive detection as $1.5 \mathrm{~dB}$ [11]. FWHM and LE are acceptable if they result in imaged dimensions comparable to actual targets dimensions. MMR is the ratio between the Maximum Magnitude (MM) of the image where refraction is not considered and the MM of the corresponding image when refraction is considered. MMR equal to 1 means there is no change in magnitude between the images. DE is the average difference between the full electrical distance $\left(d_{a i}+n_{d i e l} d_{i v}\right)$ between each antenna position $a$ and each voxel $v$ when considering or not considering refraction. DE can be compared to the theoretical range resolution of the imaging system $\Delta d=\frac{c}{4 n_{\text {diel }} \Delta f}$ where $\Delta f$ is the frequency bandwidth.

\section{EVALUATION OF REFRACTION IN REGULAR SHAPES}

This section describes the numerical setup used to evaluate the refraction effects using a regular shape: a simplified spherical phantom. All elements were simulated using Computer Simulation Technology (CST) Microwave Studio software [12]. The refracted rays paths were computed analytically.

\section{A. Numerical setup}

As a simplified version of the body part (which could represent the breast or the head) we considered a $50 \mathrm{~mm}$ radius homogeneous dielectric sphere with a complex permittivity of $\epsilon_{c}=\epsilon_{r}-j 0.1 \epsilon_{r}$. Two PEC spheres with radius $r_{t}$ were used as targets and embedded in the phantom at coordinates $(x, y, z)=(-10,30,0)$ and $(20,-20,0) \mathrm{mm}$, respectively.

We simulated a monostatic system based on a planar slotbased single-layer printed antenna formed by two crossed exponential slots (in short, XETS) [13]. It is impedancematched from 2 to $6 \mathrm{GHz}$ [9], covering the frequency band commonly used for medical MWI applications [1], [14]. The XETS swept the spherical phantom across an $80 \mathrm{~mm}$ radius circumference (i.e. $30 \mathrm{~mm}$ away from the sphere surface) contained in the plane $z=0 \mathrm{~mm}$ in a total of $N_{a}$ positions, equidistant, with an angular step of $\frac{360^{\circ}}{N_{a}}$.

\section{B. Analysis of refraction in imaging results}

In the following sub-sections, we discuss the influence of the refraction effects on the imaging results for variations of three parameters: average relative permittivity of the phantom $\left(\epsilon_{r}=\right.$ $4,8,20,40)$, targets radius $\left(r_{t}=2.5,5,7.5 \mathrm{~mm}\right)$, and number of antennas $\left(N_{a}=8,12,16\right)$. The average relative permittivity values are chosen to represent a fatty breast $\left(\epsilon_{r}=4\right.$ or 8$)$ and head tissues $\left(\epsilon_{r}=40\right)$, and an intermediate value $\left(\epsilon_{r}=\right.$ $20)$. Analytical calculations in the spherical breast scenario showed that the inclusion of refraction increased the image reconstruction time about 84 -fold. Due to space constraints, we only present the most representative images obtained for a subset of the tests, but conclusions are drawn from all results.

1) Relative permittivity: Fig. 2 summarises the resulting metrics calculated for all considered average relative permittivity values (with fixed $r_{t}=5 \mathrm{~mm}$ and $N_{a}=16$ ). As illustrated in Fig. 3(a,b), for low permittivity values $\left(\epsilon_{r}=4\right)$ refraction calculation may be discarded, as the target is detected correctly in both cases. MMR is lower than 1, thus showing better energy focusing, but SCR is comparable between when refraction is or is not considered, as shown in Fig. 2. Both FWHM and LE are within the targets size (10 mm diameter) and do not change substantially when refraction is considered. Similar behaviour is observed when $\epsilon_{r}=8$. The images when refraction is or is not considered are also visually similar.

When $\epsilon_{r}$ increases, refraction calculation becomes more relevant and should be considered in image reconstruction. We observe in Fig. 3(c,d) that, for $\epsilon_{r}=40$, the image is better focused when we consider refraction, which results in MMR lower than 0.5. Additionally, SCR indicates that the targets detection is only possible when refraction is considered. Moreover, FWHM increases when considering refraction, which is

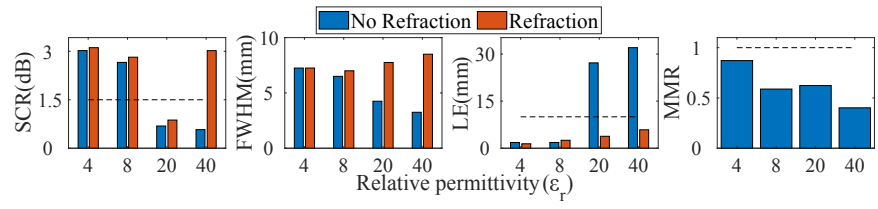

Fig. 2. Performance metrics varying the relative permittivity value $\left(\epsilon_{r}=\right.$ $4,8,20,40)$ of the spherical phantom $\left(r_{t}=5 \mathrm{~mm}\right.$ and $\left.N_{a}=16\right)$. 


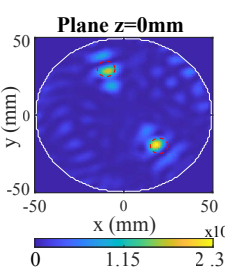

(a)

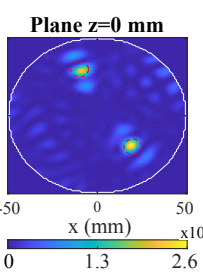

(b)

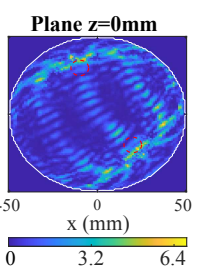

(c)

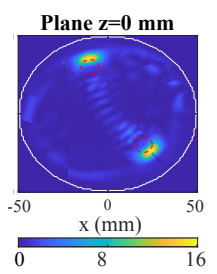

(d)
Fig. 3. Reconstructed images in $x y$-plane of spherical phantom with (a,b) $\epsilon_{r}=4$ and $(\mathrm{c}, \mathrm{d}) \epsilon_{r}=40$ when $(\mathrm{a}, \mathrm{c})$ not considering and $(\mathrm{b}, \mathrm{d})$ considering refraction. The red dashed circles represent the targets true location and size.

a result of the focusing. LE decreases when considering refraction, with both targets detected correctly. Using a phantom of $\epsilon_{r}=20$, FWHM and LE behaviour is similar when refraction is considered which indicates that targets are focused and detected in the correct location. However, low SCR, even when refraction is considered, is explained by high levels of clutter, which means the identification of the targets is not reliable.

$\mathrm{DE}$ is $3.1,11.9,23.7$ and $38.9 \mathrm{~mm}$ for $\epsilon_{r}=4,8,20$ and 40 , respectively. These values illustrate a large difference in the computation of distances when refraction is or is not considered for higher $\epsilon_{r}$. Moreover, the range resolution $\Delta d$ is 9.4, 6.6, 4.2 and $3.0 \mathrm{~mm}$ for $\epsilon_{r}=4,8,20$ and 40 , respectively. DE is not substantially higher than $\Delta d$ for lower $\epsilon_{r}$, which corroborates refraction might be disregarded in these conditions. For higher $\epsilon_{r}$, the computation of refraction has a clear impact on imaging results and needs to be computed.

2) Targets size: The results hereby discussed were obtained using only $\epsilon_{r}=4$ and 40 , and $N_{a}=16$. We assumed two additional target sizes of 2.5 and $7.5 \mathrm{~mm}$ in radius.

As shown in Fig. 4, the impact of computing refraction on SCR and FWHM is negligible, regardless the size of the targets embedded in the phantom, for both $\epsilon_{r}$. LE behaviour only changes for a particular test where $\epsilon_{r}=40$ and $r_{t}=7.5 \mathrm{~mm}$. In this test, LE is within the size of the targets whether refraction is or is not considered. Nonetheless, refraction is relevant to ensure the target is detected with high SCR and a FWHM within the targets dimensions. Targets size does not affect DE. These results are relevant, as they demonstrate that the influence of refraction on the targets detection in both low and high relative permittivity media is independent of targets size, which is unknown in most cases.

3) Number of antennas: The results discussed in this point were obtained using $\epsilon_{r}=4$ and 40 and $r_{t}=5 \mathrm{~mm}$, and are presented in Fig. 5. We assumed three different sets of antennas for comparison: $N_{a}=8,12$ and 16. When

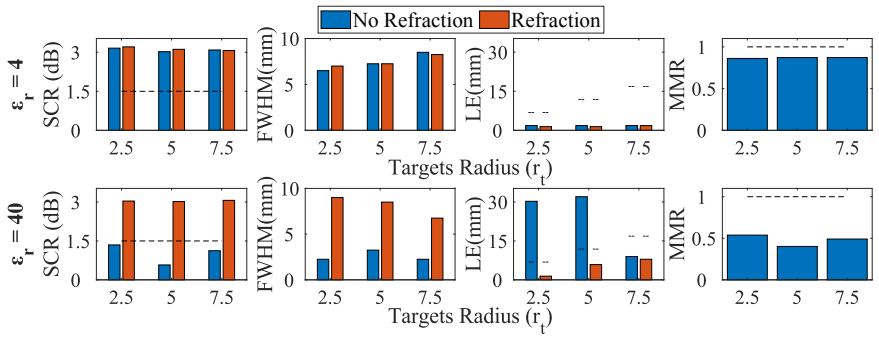

Fig. 4. Performance metrics varying the targets radius $\left(r_{t}=2.5,5,7.5\right)$ embedded in the spherical phantom $\left(\epsilon_{r}=4,40\right.$ and $\left.N_{a}=16\right)$.

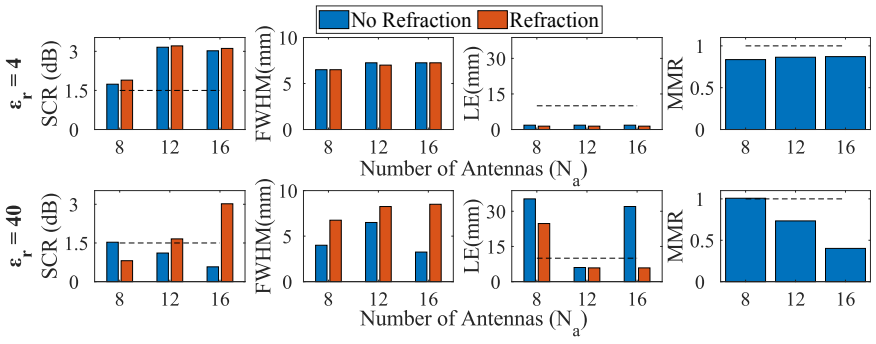

Fig. 5. Performance metrics varying the number of antennas used to obtain the images $\left(N_{a}=8,12,16\right)$ of the phantom $\left(\epsilon_{r}=4,40\right.$ and $\left.r_{t}=5 \mathrm{~mm}\right)$.

$\epsilon_{r}=4$, refraction does not impact imaging results regardless the number of antennas used. When $\epsilon_{r}=40$, refraction is important to improve imaging results for both $N_{a}=16$ and 12. SCR increases in both cases when refraction is considered which results in a SCR higher than $1.5 \mathrm{~dB}$. For lower $N_{a}$, the targets are not detected whether refraction is considered or not. We demonstrate that the targets detection in an image is very sensitive to the number of antennas and their positioning, in particular a too low number of antennas fails to result in the correct targets detection. Varying $N_{a}$ also does not affect DE.

In summary, refraction effects are important to be considered in high permittivity media regardless of targets size. Furthermore, our results corroborate that one should use the largest number of antennas as possible around the phantom, to ensure better focusing of the image. Refraction computation does not substantially improve the imaging results when a low number of antennas is used. In situations of low average permittivity within the phantom, independently of the targets size and number of antennas used, the improvement of imaging results when considering refraction are not significant enough to justify the associated increase of computational cost.

\section{VALIDATION WITH ANTHROPOMORPHIC PHANTOMS}

In this section, we present the validation of previous results with realistic-shaped phantoms. To this end, we use the ray tracing algorithm described in section II. Refraction effects are studied in monostatic setups with a breast phantom simulated using CST and an axillary region phantom using an experimental prototype (Fig. 6). While the breast is mostly convex, the axillary region has both concave and convex surfaces. The computational time when including refraction with the ray tracing algorithm, increased by 10 to 131 -fold, according to the shape complexity and the size of the imaged region. Fig. 7 summarises the performance metrics with both setups.

\section{A. Anthropomorphic breast phantom}

We considered a Magnetic Resonance Imaging-derived breast phantom from the University of Wisconsin-Madison repository (ID: 062204) [15] with a homogeneous medium of $\epsilon_{r}=8$ and loss tangent of 0.1. A PEC target was embedded in the phantom at coordinates $(x, y, z)=(25,0,-30) \mathrm{mm}$. The XETS swept the breast in the $y=0 \mathrm{~mm}$ plane with a radius of $80 \mathrm{~mm}$ from the phantom centre in 7 equally-spaced positions (angular step of $30^{\circ}$ ), as shown in Fig. 6a.

Fig. 8 shows the imaging results of an axial plane of the breast, with antenna positions marked in magenta. DE is 


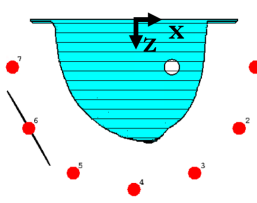

(a)

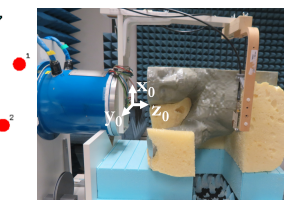

(b)

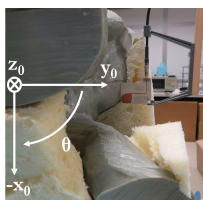

(c)
Fig. 6. (a) Numerical setup of the anthropomorphic breast and experimental setup with anthropomorphic axillary region in (b) coronal and (c) axial views.

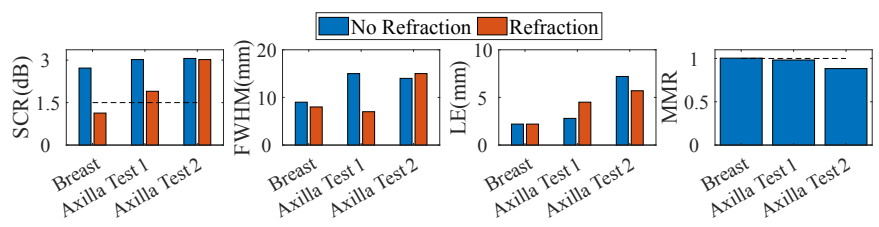

Fig. 7. Performance metrics with anthropomorphic phantoms.

$7.9 \mathrm{~mm}$ which is slightly higher than $\Delta d(6.6 \mathrm{~mm}) . \mathrm{MMR}$ is 1 , and SCR and FWHM decrease when considering refraction. However, Fig. 8 shows no relevant artefacts in the resulting image with refraction outside the region of the target. Lastly, the target is correctly detected, with LE within the target dimensions in both cases. Thus, our results indicate there is no clear advantage of considering refraction when imaging a realistic breast phantom with the current characteristics, which agrees with the results presented for the spherical phantom.

\section{B. Anthropomorphic axillary region phantom}

The axillary region phantom is a 3D-printed model segmented from a Computed Tomography image (Fig. 6b-c). It comprises a cavity where a 3D-printed model of an Axillary Lymph Node (ALN) - an ellipsoid with $20 \times 13 \times 11 \mathrm{~mm}^{3}$ - was placed in two different positions in different experimental tests. Both axillary region and ALN phantoms were filled with liquid mixtures [16] in order to mimic the dielectric properties of fat and ALNs, respectively. At $4 \mathrm{GHz}$, the dielectric properties were $\epsilon_{r}=4$ and 55 and $\sigma=0.01$ and $3.5 \mathrm{~S} / \mathrm{m}$ for fat and ALN tissues, respectively. A Vivaldi antenna (presented in [17]) was swept in a total of 9 positions (angular step of $\left.10^{\circ}\right)$ in the same $x y$-plane as the target. The full description of this setup is provided in [18]. The same frequency band $(2-6 \mathrm{GHz})$ was considered for image reconstruction.

Fig. 9 shows the imaging results of two targets placed in different $x y$-planes of the axillary phantom, which correspond to axial planes of the body. The results in both planes are similar, even though the axillary region profile is quite different between these planes. The target is correctly detected whether refraction is or is not considered. DE is 6.2 and $12.1 \mathrm{~mm}$ for test 1 and 2, respectively, which are in same order of magnitude of $\Delta d(9.4 \mathrm{~mm})$. Both LE and FWHM are within the target dimensions in all cases. SCR and FWHM decrease when refraction is considered and MMR is close to 1 in test 1. Nonetheless, the target detection is still satisfactory, and no relevant artefacts are visually observed. In test 2 , no substantial differences are observed whether refraction is considered or not. Despite the complex shape of both considered planes, the refraction effects on the imaging results are negligible.

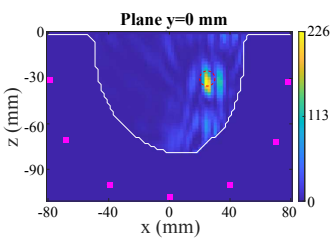

(a)

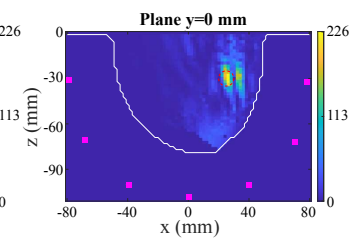

(b)
Fig. 8. Reconstructed images in $x z$-plane of realistic breast phantom with $\epsilon_{r}=8$ when (a) not considering and (b) considering refraction. The red dashed circle represents the true location and size of the target, and the magenta points represent the antenna positions surrounding the breast.

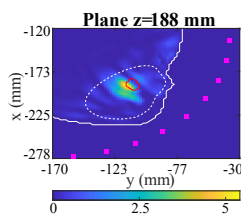

(a)

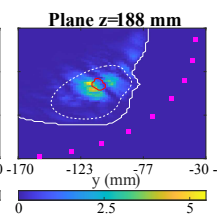

(b)

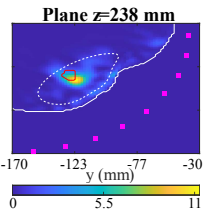

(c)

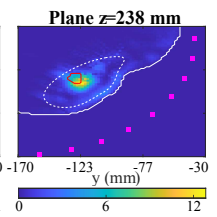

(d)
Fig. 9. Reconstructed images in $x y$-plane of test $1(\mathrm{a}, \mathrm{b})$ and test 2 (c,d) using an axillary region phantom when $(\mathrm{a}, \mathrm{c})$ not considering and $(\mathrm{b}, \mathrm{d})$ considering refraction. The red dashed circle represents the true location and size of the target, the white dashed contour represents the region where the lymph nodes are likely to be located and the magenta points represent the antenna positions.

\section{CONCLUSION}

The study aimed to assess the importance of considering refraction in MWI image reconstruction algorithms, which increases computational time, especially using dry setups where the absence of a contact liquid enhances refraction.

The study was based on full wave simulations, as well as experimental measurements. We used a spherical dielectric as an idealised model of a body part like the breast or the head, as well as anthropomorphic models of the breast and the axillary region. The first model allowed studying the effect of different values of the phantom average permittivity, targets size and number of antennas, while the latter, with all the influence of a complex body shape and a real setup in the case of the axillary region model, allowed validating the conclusions with realistic data. Conclusions of the high permittivity of the body were also validated with a simulated anthropomorphic model of the head with $\epsilon_{r}=40$ (data not shown due to space constraints). The study assessment used performance metrics as MMR, SCR, and LE.

We demonstrated that it is acceptable to discard refraction for low to moderate values of average $\epsilon_{r}$ of the body $\left(\epsilon_{r} \leq 8\right)$ : it may reduce MMR but it does not affect significantly the image quality, yielding high SCR and low LE. Conversely, for $\epsilon_{r}>8$ (e.g. MWI for head imaging), imaging results may deteriorate, causing clutter and artefacts. These effects aggravate as the number of antennas is reduced. We observed that targets size does not influence the impact of refraction.

The described variety of factors considered in the study, including the simulated and experimental validation for very irregular shapes where refraction is enhanced, ensures that the above conclusions can be generally considered. The above guidelines shall be used for researchers to decide when to invest computational time on the evaluation of refraction paths instead of straight paths, especially using dry setups. 


\section{ACKNOWLEDGMENT}

The authors would like to thank António Almeida for his assistance in the measurements.

\section{REFERENCES}

[1] J. M. Felício, J. M. Bioucas-Dias, J. R. Costa, and C. A. Fernandes, "Microwave Breast Imaging using a Dry Setup," IEEE Trans. Comput. Imag., vol. 6, pp. 167-180, 2020.

[2] D. M. Godinho, J. M. Felício, C. A. Fernandes, and R. C. Conceição, "Feasibility study of focal lens for multistatic microwave breast imaging," in Proc. $23^{\text {rd }}$ Int. Conf. Appl. Electromagn. and Commun. (ICECOM), Dubrovnik, Croatia, 2019, pp. 1-5.

[3] M. T. Islam, M. Z. Mahmud, M. T. Islam, S. Kibria, and M. Samsuzzaman, "A Low Cost and Portable Microwave Imaging System for Breast Tumor Detection Using UWB Directional Antenna array," Sci. Rep., vol. 9, pp. 15 491-15 504, 2019.

[4] A. Vispa et al., "UWB device for breast microwave imaging: phantom and clinical validations," Measurement, vol. 146, pp. 582-589, 2019.

[5] T. Reimer, M. Solis-Nepote, and S. Pistorius, "The application of an iterative structure to the delay-and-sum and the delay-multiply-and-sum beamformers in breast microwave imaging," Diagnostics, vol. 10, no. 6, pp. 411-426, 2020.

[6] A. T. Mobashsher and A. M. Abbosh, "On-site Rapid Diagnosis of Intracranial Hematoma using Portable Multi-slice Microwave Imaging System," Sci. Rep., vol. 6, pp. 37 620-37 637, 2016.

[7] Y. Wang and A. Abbosh, "Three-dimensional Microwave Breast Imaging Using Least Electrical Path Method," in Proc. Asia-Pacific Microw. Conf. (APMC), Seoul, South Korea, 2013, pp. 1021-1023.

[8] W. Shao, S. Member, T. R. Mccollough, and W. J. Mccollough, "A Phase Shift and Sum Method for UWB Radar Imaging in Dispersive Media," IEEE Trans. Microw. Theory Techn., vol. 67, no. 5, pp. 20182027, 2019.

[9] D. M. Godinho, J. M. Felício, C. A. Fernandes, and R. C. Conceição, "Study of the Refraction Effects in Microwave Breast Imaging Using a Dry Setup," in Proc. $42^{\text {nd }}$ Annu. Int. Conf. IEEE Eng. Med. Biol. Soc. (EMBC), Montreal, Canada, 2020, pp. 1787-1790.

[10] J. M. Lopez-Sanchez and J. Fortuny-Guasch, "3-D Radar Imaging Using Range Migration Techniques," IEEE Trans. Antennas Propag., vol. 48, no. 5, pp. 728-737, 2000.

[11] D. O'Loughlin, "Improving the Sensitivity of Radar-based Breast Imaging Algorithms in Diverse Patient Populations," Ph.D. dissertation, National University of Ireland Galway, 2018.

[12] Dassault Systèmes, "CST - Computer Simulation Technology," 2020. [Online]. Available: https://www.3ds.com/productsservices/simulia/products/cst-studio-suite/

[13] J. R. Costa and C. A. Fernandes, "Broadband slot feed for integrated lens antennas," IEEE Antennas Wireless Propag. Lett., vol. 6, pp. 396-400, 2007.

[14] A. S. Alqadami, N. Nguyen-Trong, B. Mohammed, A. E. Stancombe, M. T. Heitzmann, and A. Abbosh, "Compact Unidirectional Conformal Antenna Based on Flexible High-Permittivity Custom-Made Substrate for Wearable Wideband Electromagnetic Head Imaging System," IEEE Trans. Antennas Propag., vol. 68, no. 1, pp. 183-194, 2020.

[15] M. J. Burfeindt et al., "MRI-Derived 3-D-Printed Breast Phantom for Microwave Breast Imaging Validation," IEEE Antennas Wireless Propag. Lett., vol. 11, pp. 1610-1613, 2012.

[16] N. Joachimowicz, C. Conessa, T. Henriksson, and B. Duchêne, "Breast phantoms for microwave imaging," IEEE Antennas Wireless Propag. Lett., vol. 13, pp. 1333-1336, 2014.

[17] J. M. Felicio, J. M. Bioucas-Dias, J. R. Costa, and C. A. Fernandes, "Antenna design and near-field characterization for medical microwave imaging applications," IEEE Trans. Antennas Propag., vol. 67, no. 7, pp. 4811-4824, 2019.

[18] D. M. Godinho, J. M. Felício, C. A. Fernandes, and R. C. Conceição, "Experimental Evaluation of an Axillary Microwave Imaging System to Aid Breast Cancer Staging," unpublished. 